\title{
Safety of Eslicarbazepine Acetate in Elderly Versus Non-Elderly Patients with Focal Seizures: From Pooled Data of Clinical Studies to 8 Years of Post-Marketing Experience
}

\author{
Luís M. Magalhães $^{1} \cdot$ Raquel Costa $^{1} \cdot$ Mariana Vieira $^{1} \cdot$ Joana Moreira ${ }^{1} \cdot$ Helena Gama ${ }^{1} \cdot$ Patrício Soares-da-Silva $^{1,2,3} \mathbb{D}^{0}$
}

Accepted: 5 July 2021 / Published online: 18 September 2021

(C) The Author(s) 2021

\begin{abstract}
Introduction The prevalence of epilepsy increases in elderly patients aged $>65$ years, and treatment is challenging because clinical data are limited.

Objective Our objective was to evaluate the safety of eslicarbazepine acetate (ESL) in patients aged $\geq 65$ years versus nonelderly patients with focal seizures.

Methods The safety data of seven phase II and III, double-blind, open-label, randomized clinical studies of ESL in adults were pooled. At least possibly related treatmentemergent adverse events (TEAEs) and ESL post-marketing adverse drug reactions (ADRs) were analyzed separately by age categories.

Results The most frequently reported at least possibly related TEAEs in elderly $(N=120)$ versus non-elderly patients $(N=1863)$ were dizziness (10.8 vs. $20.3 \%)$, somnolence (9.2 vs. $12.6 \%)$, and hyponatremia (6.7 vs. $1.5 \%)$. Elderly patients presented a higher incidence of serious TEAEs ( $22.5 \mathrm{vs.} 7.6 \%)$ and at least possibly related serious TEAEs (6.7 vs. $2.5 \%)$, probably because treatment was complicated by comorbidities and comedications. After an estimated cumulative exposure of over 2 million patient-months worldwide and 8 years of post-marketing surveillance, hyponatremia was the most frequently reported ADR $(n=232)$, accounting for $14.6 \%$ and $6.8 \%$ of the ADRs reported in elderly $(n=473)$ and non-elderly patients $(n=2406)$, respectively. This was followed by ADR/safety information such as drug-dose titration not performed (7.0 vs. 5.4\%), product use in unapproved indication (4.9 vs. $1.9 \%)$, off-label use (3.4 vs. $2.2 \%)$, dizziness (3.4 vs. $3.5 \%$ ), and seizure (2.1 vs. $5.8 \%)$.

Conclusion No specific safety issue was identified from the pooled studies for elderly compared with non-elderly patients. After 8 years of post-marketing surveillance, the qualitative safety of ESL remains similar to that observed in the clinical studies.
\end{abstract}

Luís M. Magalhães

luis.magalhaes@bial.com

1 Division of Research and Development, Bial-Portela $\& C^{a}$, S.A., À Avenida da Siderurgia Nacional, 4745-457 Coronado (S. Romão e S. Mamede), Portugal

2 Department of Biomedicine, Unit of Pharmacology and Therapeutics, Faculty of Medicine, University of Porto, Porto, Portugal

3 MedInUP-Center for Drug Discovery and Innovative Medicines, University of Porto, Porto, Portugal

\section{Key Points}

Safety data from the seven clinical studies indicate that no specific safety issues were identified for treatment of focal seizures with eslicarbazepine acetate (ESL) for elderly patients.

After 8 years of post-marketing surveillance, the qualitative safety of ESL was consistent with data obtained from clinical studies.

ESL was generally safe and well-tolerated in elderly patients. 


\section{Introduction}

Epilepsy affects approximately 50 million adults and children worldwide $[1,2]$. The incidence is highest in children and in elderly people aged $>65$ years $[3,4]$. The overall prevalence varies from 4 to 8 per 1000 people, increasing considerably in people aged $>65$ years [5]. Indeed, epilepsy is the most common disease in the elderly after cerebrovascular disorders and dementia [6]. With a progressive increase in life expectancy, patients aged $>65$ years constitute the fastest growing segment of patients with epilepsy [7].

The treatment of elderly patients with epilepsy is particularly challenging, as this age group is rarely represented in the clinical trials that have led to the approval of the different antiseizure medications (ASMs) [1, 8]. Treatment is complicated by the presence of physiological changes related to aging, comorbidities, cognitive problems, and concerns regarding drug interactions and medication adherence [7].

Indeed, these patients have a higher incidence of comorbidities and a higher use of concomitant medications that may result in drug-drug interactions [1, 2, 9-11], including not only interactions with other drugs but also interactions between different ASMs in a polytherapy regimen [6]. Furthermore, age-related physiological changes such as hepatic and renal impairment affect the pharmacokinetics and pharmacodynamics of ASMs and may affect the nature, incidence, and severity of adverse events (AEs) $[3,6,11]$, making these patients particularly sensitive to certain secondary effects of ASMs, such as, for example, cognitive disturbances, hyponatremia, or osteoporosis [8].

Eslicarbazepine acetate (ESL) is a once-daily ASM that is approved in Europe as monotherapy in adults with newly diagnosed epilepsy for the treatment of focal seizures (FS), with or without secondary generalization, and in adults, adolescents, and children aged $>6$ years with FS, with or without secondary generalization, as adjunctive therapy [12]. In the USA, ESL is approved in patients aged $\geq 4$ years for the treatment of FS [13]. Gama et al. [14] summarized the safety data of two phase I studies, a phase III study in elderly participants, and pooled data from four phase III studies and compared them with real-world data obtained from 6 years of post-marketing surveillance. The authors concluded that, after 6 years on the market, ESL continued to have a similar safety profile to that observed in the reported clinical studies [14]. More detailed data from the phase III study in the elderly $(N=72)$ were published separately and confirmed previously published results [15]. Recently, a subanalysis from the Euro-Esli study regarding the effectiveness and safety of ESL in patients with epilepsy aged $\geq 60$ versus $<60$ years [16] and a comparative analysis of the safety and tolerability of ESL in older ( $\geq 60$ years) and younger (18-59 years) adults was published [17]. The safety/tolerability of ESL in patients aged $\geq 60$ years was consistent with its known profile.

In this work, pooled data from seven clinical studies were used to assess the safety and tolerability of ESL in an older population of patients diagnosed with FS aged $\geq 65$ years compared with non-elderly patients (18-64 years). Furthermore, ESL safety data from 8 years of post-marketing surveillance, which is a valuable source of complementary evidence, were compared between age groups and with the clinical study data. The incidence and nature of treatmentemergent adverse events (TEAEs) from clinical studies and the type and relative frequency of most commonly reported adverse drug reactions (ADRs) from post-marketing surveillance were evaluated and compared between age groups aimed to further assess the safety of ESL in elderly patients.

\section{Methods}

\subsection{Pooled Analysis of Seven Clinical Studies}

Safety data from the following clinical studies (all registered at ClinicalTrials.gov) were pooled and analyzed by age group: BIA-2093-201 (NCT02170077) [17], BIA2093-301 (parts I-IV) (NCT00957684) [18], BIA-2093302 (parts I and II) (NCT00957047) [19], BIA-2093-303 (parts I and II) (NCT00957372) [20], BIA-2093-304 (part I) (NCT00988429) [21], BIA-2093-311 (part I) (NCT02484001) [22], and BIA-2093-401 (NCT01422720) [15]. These studies were all randomized, double-blind or open-label phase II/III clinical studies in FS; study BIA2093-303 was excluded from the US FDA new drug application for ESL because of major protocol violations that cast doubts on the reliability of the study results. The table in the electronic supplementary material (ESM) describes the study design, ESL dosages, treatment duration, and number of elderly and non-elderly patients involved in each study. Details of inclusion and exclusion criteria as well as efficacy, safety, and tolerability assessments were published previously [15, 18-23].

The safety analysis included all randomized patients who received at least one dose of study medication. Descriptive statistics were summarized using frequency and percentage of TEAEs overall and by age group (18-64 and $\geq 65$ years). TEAEs are AEs with an onset after initiation of ESL treatment or that worsened either in intensity or frequency relative to the pretreatment state and were coded using the Medical Dictionary for Regulatory Activities (MedDRA ${ }^{\circledR}$ ) version 5.0 for study $-201 ; 10.0$ for studies $-301,-302$, and $-303 ; 13.1$ for study $-304 ; 16.1$ for 
study -311; and 16.0 for study -401. TEAE assessment included intensity, causality, and seriousness. TEAEs were considered related to ESL treatment if the investigator(s) assessed these to be at least possibly related to ESL. Data were compared exploratively for elderly ( $\geq 65$ years) versus non-elderly ( $<65$ years) adult patients using the Chi-squared test or Fisher's exact test, as appropriate. A $p$ value $<0.05$ was considered statistically significant, but $p$ values should be interpreted carefully since this is an exploratory post-hoc analysis of different clinical studies with several limitations.

The studies were approved by the appropriate ethics committees or institutional review boards. They were conducted according to the Declaration of Helsinki, the International Council on Harmonization Good Clinical Practice, and local regulations. Prior to enrolment, patients gave their written informed consent.

\subsection{Analysis of Post-Marketing Surveillance Data}

The marketing authorization holder collected post-marketing safety data worldwide from spontaneous reports, health authority reports, literature reports, reports from non-interventional studies, and other solicited sources in the master safety database (Argus Safety ${ }^{\mathrm{TM}}$ ) as part of routine pharmacovigilance activities. The Argus Safety ${ }^{\mathrm{TM}}$ database was searched for all cases from post-marketing surveillance, from date of first launch on 1 October 2009 up to 21 October 2017 (8 years). ADRs were coded using MedDRA version 21.0 and assessed for seriousness and listedness. All spontaneous and literature reports were pooled and defined as having an at least possible causality; solicited reports were pooled separately and included only cases assessed as at least possibly related to ESL by the reporter.

ADRs/safety information were analyzed by age group and presented in cumulative summary tabulations by primary system organ class (SOC) and preferred terms (PTs), with PTs sorted by decreasing frequency within each SOC. Additionally, the percentage of serious ADRs reported from the total ADRs/safety information was calculated. SOCs were sorted according to the EU summary of product characteristics (SmPC) guideline. Results were compared with data in the approved SmPC.

Estimation of patient exposure was based on the assumption that the ex-factory amounts delivered were entirely dispensed and all administered as 1 tablet per day, regardless of dose strength, as recommended in the ESL SmPC [12, 13]. As ESL is intended for long-term therapy, exposure was calculated in patient-months (units divided by 30 ) and patient-years (patient-months divided by 12) rather than in number of treated patients.

\section{Results}

\subsection{Safety Findings from Pooled Analysis of Seven Clinical Studies}

In this pooled analysis of seven clinical studies, a total of 120 elderly patients ( $\geq 65$ years) and 1863 non-elderly patients (18-64 years) were treated with ESL (Table 1). Most of the elderly patients ( $n=72)$ were enrolled in study BIA-2093-401 (see the Table in the ESM). The frequency of patients with any TEAE was similar between elderly and non-elderly patients (99/120 [82.5\%] vs. 1434/1863 [77.0\%]; $p=0.1612$, Chi-squared test) as were the frequencies of at least possibly related TEAEs (62/120 [51.7\%] vs. $1015 / 1863$ [54.5\%]; $p=0.3601$, Chi-squared test). Furthermore, the frequency with which patients discontinued from the clinical studies because of TEAEs was also similar between elderly and non-elderly patients $(24 / 120$ [20.0\%] vs. 315/1863 [16.9\%]; $p=0.7889$, Chi-squared test). Serious TEAEs were reported more frequently in elderly than in non-elderly patients $(27 / 120$ [22.5\%] vs. $142 / 1863$ [7.6\%]; $p<0.0001$, Chi-squared test) as were at least possibly related serious TEAEs (8/120 [6.7\%] vs. 46/1863 [2.5\%]; $p=0.0062$, Chi-squared test) (Table 1). Discontinuations because of TEAEs in $\geq 1 \%$ of elderly patients comprised ataxia (3/24 [2.5\%]), hyponatremia (3/24 [2.5\%]), convulsion (2/24 [1.7\%]), dizziness (2/24 [1.7\%]), and fatigue (2/24 [1.7\%]). The most commonly reported TEAEs leading to discontinuation in non-elderly adults were dizziness (80/315 [4.3\%]), nausea $(45 / 315$ [2.4\%]), vomiting (39/315[2.1\%]), diplopia (29/315 [1.6\%]), and somnolence (27/315 [1.4\%]), whereas ataxia and hyponatremia were reported for $32 / 315(1.7 \%)$ and $5 / 315(0.3 \%)$ of patients, respectively [24]. The majority of TEAEs in both groups were of mild or moderate intensity. Severe TEAEs were reported for a higher percentage of elderly than non-elderly patients (22/120 [18.3\%] vs. $222 / 1863$ [11.9\%]), with the most commonly reported severe TEAEs $(\geq 1 \%)$ in elderly patients being fatigue, vertigo, and toxicity to various agents (each $2 / 120$ [1.7\%]), whereas dizziness $(42 / 1863$ [2.3\%]), headache $(26 / 1863$ [1.4\%]), ataxia (23/1863 [1.2\%]), and nausea (18/1863 [1.0\%]) were reported in $\geq 1 \%$ of non-elderly patients [24].

The most frequently reported at least possibly related TEAEs in both elderly and non-elderly patients were dizziness $(13 / 120$ [10.8\%] vs. $378 / 1863$ [20.3\%]; $p$ $=0.1990$, Chi-squared test $)$ and somnolence $(11 / 120$ [9.2\%] vs. $235 / 1863$ [12.6\%]; $p=0.1612$, Chi-squared test) (Table 2). The events hyponatremia (8/120 [6.7\%] vs. $28 / 1863$ [1.5\%], Chi-squared test), fatigue (7/120 [5.8\%] vs. 66/1863 [3.5\%]; $p=0.1965$, Chi-squared test), increased gamma-glutamyltransferase (4/120 [3.3\%] vs. 
Table 1 Pooled analysis of ESL clinical studies: summary of treatment-emergent adverse events in elderly $(\geq 65$ years) and non-elderly (18-64 years) patients

\begin{tabular}{lcc}
\hline TEAEs & $\begin{array}{c}\text { Elderly patients } \\
(N=120)\end{array}$ & $\begin{array}{c}\text { Non-elderly } \\
\text { patients } \\
(N=1863)\end{array}$ \\
\hline Patients with any TEAE & $99(82.5)$ & $1434(77.0)$ \\
Mild & $16(13.3)$ & $456(24.5)$ \\
Dizziness & $9(7.5)$ & $239(12.8)$ \\
Fatigue & $7(5.8)$ & $49(2.6)$ \\
Hypertension & $7(5.8)$ & $31(1.7)$ \\
Nasopharyngitis & $7(5.8)$ & $67(3.6)$ \\
Moderate & $49(40.8)$ & $712(38.2)$ \\
Dizziness & $7(5.8)$ & $164(8.8)$ \\
Headache & $6(5.0)$ & $112(6.0)$ \\
Somnolence & $6(5.0)$ & $90(4.8)$ \\
Severe & $22(18.3)$ & $222(11.9)$ \\
Fatigue & $2(1.7)$ & $4(0.2)$ \\
Vertigo & $2(1.7)$ & $13(0.7)$ \\
Patients with any treatment-related ${ }^{\mathrm{a}}$ TEAE $^{\mathrm{b}}$ & $62(51.7)$ & $1015(54.5)$ \\
Patients with any TEAE leading to discontinuation & $24(20.0)$ & $315(16.9)$ \\
Ataxia & $3(2.5)$ & $32(1.7)$ \\
Hyponatremia & $3(2.5)$ & $5(0.3)$ \\
Patients with any treatment-related serious TEAE & $8(6.7)$ & $46(2.5)$ \\
Hyponatremia & $2(1.7)$ & $2(0.1)$ \\
\hline
\end{tabular}

Data are presented as $n(\%)$ unless otherwise indicated

Studies: BIA-2093-201, -301 (parts I-IV), -302 (parts I and II), -303 (parts I and II), -304 (part I), -401, and -311 (part I, DLP 21-Oct-2017) combined

The most common TEAEs in the elderly population for each category is described to enable comparison with non-elderly populations

$E S L$ eslicarbazepine acetate, $N$ number of patients in group, $n$ number of patients with event, TEAE treatment-emergent adverse event

${ }^{a}$ Treatment-related TEAEs include all TEAEs for which the investigator assessed the relationship to study medication as possible, probable, or definite

${ }^{\mathrm{b}}$ Most common preferred terms are described in Table 3
$13 / 1863[0.7 \%] ; p=0.0164$, Fisher's exact test), and decreased blood sodium (3/120 [2.5\%] vs. 13/1863 [0.7\%]; $p=0.0679$, Fisher's exact test) were reported more often $(\geq 1.5 \%)$ in elderly patients. In contrast, dizziness $(13 / 120$ [10.8\%] vs. $378 / 1863$ [20.3\%]; $p=0.1990$, Chi-squared test), somnolence (11/120 [9.2\%] vs. 235/1863 [12.6\%]; $p=0.1612$, Chi-squared test), headache (7/120 [5.8\%] vs. $154 / 1863$ [8.3\%]; $p=0.5484$, Chi-squared test), nausea $(6 / 120$ [5.0\%] vs. $149 / 1863$ [8.0\%]; $p=0.2357$, Chisquared test), and diplopia (3/120 [2.5\%] vs. 126/1863 [6.8\%]; $p=0.0664$, Chi-squared test) were reported less often in elderly patients. The incidences of hyponatremia ( $p<0.0001$, Fisher's exact test) and increased gammaglutamyltransferase ( $p=0.0164$, Fisher's exact test) were reported as treatment-related TEAEs by a significantly higher frequency of elderly than non-elderly patients (Table 2). Of note, hyponatremia was reported as a treatment-related serious TEAE in $2(1.7 \%)$ of 120 elderly patients and in $2(0.1 \%)$ of 1863 non-elderly patients exposed in these seven clinical studies [24].

\subsection{Safety Findings from Post-Marketing Surveillance Data}

A total of 473 and 2406 ADRs/safety information were reported for elderly and non-elderly patients, respectively, after 8 years of post-marketing surveillance (Table 3). Based on sales data up to 21 October 2017, patient exposure to marketed ESL was estimated at 2,417,394 patient-months (201,450 patient-years), corresponding to a reporting rate of 0.12 events per 100 patient-months [24].

The most frequently reported ADRs/safety information ( $\geq 10 \%$ of all reported in elderly) in elderly patients versus non-elderly patients by SOC were injury, poisoning, and procedural complications (19.7 vs. 16.1\%), nervous system disorders (16.5 vs. $23.9 \%$ ), metabolism and 
Table 2 Comparison of incidences (\% of patients) with at least possibly related treatment-emergent adverse events by age group and preferred term

\begin{tabular}{llcc}
\hline System organ class & TEAEs (PT) & $\begin{array}{l}\text { Elderly patients } \\
(N=120)\end{array}$ & $\begin{array}{l}\text { Non-elderly } \\
\text { patients } \\
(N=1863)\end{array}$ \\
\hline Nervous system disorders & Dizziness & $13(10.8)$ & $378(20.3)$ \\
& Somnolence & $11(9.2)$ & $235(12.6)$ \\
& Headache & $7(5.8)$ & $154(8.3)$ \\
& Ataxia & $5(4.2)$ & $69(3.7)$ \\
Metabolism and nutrition disorders & Hyponatremia & $8(6.7)$ & $28(1.5)$ \\
& Decreased blood sodium & $3(2.5)$ & $13(0.7)$ \\
General disorders and administration- & Fatigue & $7(5.8)$ & $66(3.5)$ \\
site conditions & & & $149(8.0)$ \\
Gastrointestinal disorders & Nausea & $6(5.0)$ & $13(0.7)$ \\
Investigations & Increased GGT & $4(3.3)$ & $65(3.5)$ \\
Ear and labyrinth disorders & Vertigo & $3(2.5)$ & $126(6.8)$ \\
Eye disorders & Diplopia & $3(2.5)$ & \\
\hline
\end{tabular}

Data are presented as $n(\%)$ unless otherwise indicated

Studies: BIA-2093-201, -301 (parts I-IV), -302 (parts I and II), -303 (part I and II), -304 (part I), -401, and -311 (part I, DLP 21-Oct-2017) combined. PT listed by its incidence in elderly patients (in at least 2.0\%)

$G G T$ gamma-glutamyltransferase, $N$ number of patients in group, $n$ number of patients with event, $P T$ preferred term, TEAE treatment-emergent adverse event nutrition disorders (15.4 vs. 8.0\%), general disorders and administration-site conditions (10.4 vs. $12.5 \%$ ), and skin and subcutaneous tissue disorders (10.4 vs. 7.0\%). More ADRs/safety information were reported in elderly patients than in non-elderly patients in the SOCs metabolism and nutrition disorders and psychiatric disorders; in contrast, ADRs in the SOC nervous system disorders and gastrointestinal disorders were reported more often in non-elderly than in elderly patients. The most frequently reported ADRs/ safety information ( $\geq 2 \%$ of total ADRs in elderly) by PT in elderly versus non-elderly patients were hyponatremia ( 14.6 vs. $6.8 \%$ ), drug dose titration not performed (7.0 vs. $5.4 \%$ ), product use in unapproved indication (4.9 vs. $1.9 \%$ ), off-label use (3.4 vs. $2.2 \%$ ), dizziness (3.4 vs. $3.5 \%$ ), nausea (2.3 vs. $1.5 \%)$, and seizure (2.1 vs. $5.8 \%$ ). ADRs reported with a higher percentage of total ADRs for elderly versus non-elderly patients were hyponatremia, nausea, fall, confusional state, cognitive disorder, tremor, malaise, and pruritus (Table 3). The percentage of reported ADRs/safety information that were serious was $42.3 \%$ and $31.9 \%$ for elderly and non-elderly patients, respectively [24]. Furthermore, the ADRs hyponatremia/decreased blood sodium represented $14.6 \% / 1.3 \%$ and $6.8 \% / 1.5 \%$ of ADRs reported in elderly and non-elderly patients, respectively (Table 3 ).

\section{Discussion}

Randomized, placebo-controlled clinical studies in the elderly population failed to demonstrate a clear evidence of superiority with active comparator, leading to an absence of clear-cut differences of efficacy for different ASMs in elderly patients. Therefore, treatment decisions must be driven by the drug safety profile and the individual patient's tolerability and comorbidities [25, 26].

Data from seven pooled clinical studies and from 8 years of post-marketing experience were used to assess the safety of ESL in elderly patients with FS aged $\geq 65$ years. This is particularly relevant as clinical data for the elderly population are limited and may show reduced tolerance to ASMs because of age-related physiological changes affecting the pharmacodynamics and pharmacokinetics of ASMs and drug interactions. Furthermore, the post-marketing data may provide additional insight into the use of ESL in patients with FS in a real-life setting.

The pooled clinical data indicated that, qualitatively, the safety data for ESL in elderly patients were generally similar to those observed in non-elderly patients. TEAEs were experienced at similar frequencies by elderly and nonelderly patients (99/120 [82.5\%] vs. 1434/1863 [77.0\%]). An integrated analysis of four of the seven studies included here showed a dose-dependent increase in TEAE incidence [14]. Similar frequencies of TEAEs have been reported for elderly patients receiving oxcarbazepine (42/52 [81\%]) [3] and zonisamide (78/95 [82\%]) [11]. However, both the oxcarbazepine and zonisamide analyses included fewer patients aged $\geq 65$ years than did this analysis (52 and 95, respectively, vs. 120) [3, 11]. In contrast, published results of the EuroEsli study, a pooled analysis of 14 studies, showed that significantly more elderly patients receiving ESL experienced AEs than non-elderly; however, elderly patients were defined as being aged $\geq 60$ years [16]. Consistent with previously 
Table 3 Adverse drug reactions and safety information from post-marketing data sources reported from 1 October 2009 until 21 October 2017 (with absolute frequency $\geq 5$ and $\geq 25$ for elderly and non-elderly age groups)

\begin{tabular}{|c|c|c|c|c|}
\hline \multirow[t]{2}{*}{ ADRs and safety information } & \multicolumn{2}{|c|}{$\begin{array}{l}\text { Elderly (aged } \geq 65 \\
\text { years) }\end{array}$} & \multicolumn{2}{|c|}{$\begin{array}{l}\text { Non-elderly (18-64 } \\
\text { years) }\end{array}$} \\
\hline & $\begin{array}{l}\text { Reported } \\
\text { ADRs } \\
(N=473)\end{array}$ & ADRs $(\%)$ & $\begin{array}{l}\text { Reported } \\
\text { ADRs } \\
(N=2406)\end{array}$ & ADRs $(\%)$ \\
\hline Injury, poisoning and procedural complications & $93^{\mathrm{a}}$ & 19.7 & $387^{\mathrm{a}}$ & 16.1 \\
\hline Drug dose titration not performed ${ }^{\mathrm{b}}$ & 33 & 7.0 & 129 & 5.4 \\
\hline Product use in unapproved indication ${ }^{\mathrm{b}}$ & 23 & 4.9 & 45 & 1.9 \\
\hline Off-label use $\mathrm{b}^{\mathrm{b}}$ & 16 & 3.4 & 53 & 2.2 \\
\hline Fall & 8 & 1.7 & c & c \\
\hline Inappropriate schedule of drug administration ${ }^{\mathrm{b}}$ & d & d & 36 & 1.5 \\
\hline Overdose $^{\mathrm{b}}$ & d & d & 29 & 1.2 \\
\hline Nervous system disorders & $78^{\mathrm{a}}$ & 16.5 & $574^{\mathrm{a}}$ & 23.9 \\
\hline Dizziness & 16 & 3.4 & 84 & 3.5 \\
\hline Seizure & 10 & 2.1 & 139 & 5.8 \\
\hline Somnolence & d & $\mathrm{d}$ & 43 & 1.8 \\
\hline Cognitive disorder & 6 & 1.3 & c & c \\
\hline Tremor & 5 & 1.1 & c & c \\
\hline Epilepsy & d & d & 27 & 1.1 \\
\hline Headache & d & d & 35 & 1.5 \\
\hline Metabolism and nutrition disorders & $73^{\mathrm{a}}$ & 15.4 & $193^{\mathrm{a}}$ & 8.0 \\
\hline Hyponatremia & 69 & 14.6 & 163 & 6.8 \\
\hline General disorders and administration-site conditions & $49^{\mathrm{a}}$ & 10.4 & $301^{\mathrm{a}}$ & 12.5 \\
\hline Fatigue & 6 & 1.3 & 46 & 1.9 \\
\hline Malaise & 5 & 1.1 & c & $\mathrm{c}$ \\
\hline Drug ineffective ${ }^{b}$ & d & d & 31 & 1.3 \\
\hline Skin and subcutaneous tissue disorders & $49^{\mathrm{a}}$ & 10.4 & $168^{\mathrm{a}}$ & 7.0 \\
\hline Rash & 6 & 1.3 & 45 & 1.9 \\
\hline Pruritus & 5 & 1.1 & c & c \\
\hline Gastrointestinal disorders & $34^{\mathrm{a}}$ & 7.2 & $140^{\mathrm{a}}$ & 29.6 \\
\hline Nausea & 11 & 2.3 & 35 & 1.5 \\
\hline Vomiting & 5 & 1.1 & 23 & 1.0 \\
\hline Psychiatric disorders & $21^{\mathrm{a}}$ & 4.4 & c & c \\
\hline Confusional state & 7 & 1.5 & $\mathrm{c}$ & c \\
\hline Investigations & $14^{\mathrm{a}}$ & 3.0 & $109^{\mathrm{a}}$ & 4.5 \\
\hline Decreased blood sodium & 6 & 1.3 & 37 & 1.5 \\
\hline
\end{tabular}

$A D R$ adverse drug reaction, $N$ number of ADRs

${ }^{a}$ Total numbers do not add up as they include all ADRs reported and not only those related to the preferred terms listed in the table, which were selected based on the absolute frequency $\geq 5$ and $\geq 25$ for elderly and non-elderly groups, respectively; only system organ class referring to an ADR with individual preferred term $\geq 5$ and $\geq 25$ is included, respectively

${ }^{\mathrm{b}}$ Frequency of ADRs reported were $<5$, corresponding to percentage $<1.0$

${ }^{\mathrm{c}}$ Frequency of ADRs reported were $<25$, corresponding to percentage $<1.0$

${ }^{\mathrm{d}}$ Safety information reported results of the pooled clinical studies BIA-2093301, -302, -303, -304, and -401 [14], this post-hoc analysis showed that ESL treatment resulted in a slightly higher rate of treatment discontinuation because of TEAEs in elderly than in non-elderly patients (24/120 [20.0\%] vs. $315 / 1863$ [16.9\%]); however, the Euro-Esli study, a pooled case series, showed comparable frequencies between patients aged $\geq 60$ years and those aged $<60$ years [16]. Moreover, ataxia and hyponatremia were the most common TEAE leading to discontinuation in elderly patients (3/24 [2.5\%], each) compared with non-elderly (32/315 [1.7\%] and 5/315 [0.3\%], respectively); comparable results were reported for the Euro-Esli study [14]. Dizziness (80/315 [4.3\%]) was the most common TEAE for non-elderly patients who 
discontinued ESL treatment compared with 2/24 (1.7\%) observed in elderly patients. Similar frequencies of treatment discontinuations were reported from pooled clinical studies for oxcarbazepine (14/52 [26.9\%] in elderly vs. $341 / 1574$ [21.7\%] in non-elderly) [3] and for zonisamide (17/95 [18\%] vs. 312/1389 [23\%]) [11]. Other publications have shown withdrawal rates for oxcarbazepine ranging from 12 to $67 \%$ [24]. A real-life study with ESL in elderly patients aged $>60$ years performed in Spain (ESLIBASE) showed that discontinuations because of TEAEs were more prevalent early on than late in the course of the study, indicating that severe events may occur shortly after treatment begins [10]. A study in elderly patients ( $\geq 65$ years) receiving levetiracetam as adjunctive therapy showed low frequencies of patients experiencing AEs: 53/491 (10.8\%) experienced at least one AE, 19/491 (3.9\%) experienced AEs classified as serious, and 18/491 (3.7\%) experienced AEs leading to discontinuation. These frequencies were lower than those reported for ESL; however, the levetiracetam study was a 1 -year observational study in contrast to the pooled data from randomized, double-blind, placebo-controlled studies analyzed here [27].

About half of the patients in both age groups experienced TEAEs at least possibly related to ESL treatment, mostly dizziness and somnolence. These results are consistent with those in previously reported pooled phase III studies and the open-label, non-controlled study in elderly patients [14, 15]. Moreover, similar results were reported for a pooled analysis of clinical practice studies with 2031 patients receiving ESL [27].

Furthermore, a difference in the incidence of serious TEAEs was recorded in elderly versus non-elderly patients $(27 / 120$ [22.5\%] vs. $142 / 1863$ [7.6\%]) as well as in at least possibly related serious TEAEs (8/120 [6.7\%] vs. 46/1863 [2.5\%]), which is not unexpected considering the increased comorbidities and comedications in elderly patients.

This post-hoc safety analysis reported TEAEs of hyponatremia in $8 / 120(6.7 \%)$ of the elderly patients and $28 / 1863(1.5 \%)$ of the non-elderly patients taking ESL in the seven pooled clinical studies (Table 2). Similar results were reported in the Euro-Esli study, with 19/353 (5.6\%) of patients aged $\geq 60$ years experiencing hyponatremia compared with 49/1677 (3.0\%) of patients aged $<60$ years [16]. After 8 years of post-marketing surveillance, hyponatremia and decreased blood sodium represented $14.6 \%$ (69/473 ADRs) and 1.3\% (6/473 ADRs), respectively, of all reported ADRs in elderly patients. A previous analysis by cases of hyponatremia/decreased blood sodium showed that about half of the reported cases in both elderly and non-elderly patients had blood sodium levels in the range of 120-129 $\mathrm{mmol} / \mathrm{L}$, and most of these occurred at ESL doses of 400-1200 mg [24]. In contrast, in non-elderly patients, the serious cases occurred at ESL doses > $1200 \mathrm{mg}$ [24]. The increased incidence of hyponatremia in elderly patients may be explained by the more frequent use of diuretics in elderly patients $(>20 \%)$ than in non-elderly patients $(<5.8 \%)$ because of comorbidities such as renal impairment [26]. Dose adjustments for patients experiencing hyponatremia are not considered necessary unless renal function is disturbed, although it is good clinical practice to monitor for the potential development of hyponatremia, particularly in the elderly population [28]. A phase I study demonstrated that patients with renal impairment require dose adjustments of ESL when creatinine clearance is $<60 \mathrm{~mL} / \mathrm{min}$, and ESL is not recommended if creatinine clearance is $<30 \mathrm{~mL} / \mathrm{min}$ [14].

Rash is a common reaction with ASMs, and in this post-hoc safety analysis the proportion of reported ADRs decreased. Compared with the 6-year post-marketing safety data published by Gama et al. [14], the proportion of reported rash as ADR in the 8-year post-marketing data decreased from overall $3.8 \%$ to approximately $1.8 \%$ (6/473 [1.3\%]) of ADRs in elderly and 45/2406 [1.9\%] in nonelderly patients) [14]. In the seven pooled clinical studies, rash considered related to ESL treatment occurred in $<2 \%$ of patients. In the clinical study BIA-2093-401 with patients aged $\geq 65$ years, rash was only reported in $2 / 72$ patients $(2.8 \%)$ and was considered neither severe nor serious [15].

Some ASMs have been associated with an impact on psychiatric and behavioral side effects [29]. The 8-year postmarketing surveillance data showed that psychiatric disorders had a greater proportion of all reported ADRs in elderly patients than in non-elderly patients (21/473 [4.4\%] vs. $<25 / 2406$ [ $<1.0 \%]$ of ADRs), with confusion states being the most frequent (7/473 [1.5\%] of ADRs). Age-related issues such as increased comorbidities in elderly patients (neurodegenerative disorders and cerebrovascular diseases) may have contributed to these differences [1]. Additionally, a retrospective study assessing the psychiatric profile of ESL in patients who stopped levetiracetam treatment because of psychiatric and behavioral side effects showed that ESL did not cause significantly more psychiatric side effects than in patients who had not been withdrawn from levetiracetam treatment because of these events [29].

Even though hyponatremia and decreased blood sodium occurred more frequently in elderly than non-elderly patients, the post-marketing surveillance data indicated that the qualitative safety of ESL in elderly patients was generally similar to that observed in non-elderly patients.

It is important to note that this exploratory post-hoc analysis performed in pooled clinical data has several limitations because of the small number of elderly patients $(N=120)$ compared with non-elderly patients $(N=1863)$, the inclusion of data from double-blind and open-label extension studies, the differing durations of exposure and doses of ESL, the differing therapeutic regimens 
(monotherapy and polytherapy), and the differing study designs (titration phase, treatment duration, etc.). Results of this study are likely to represent an underestimation of the side effects given that even elderly patients with multiple comorbidities were usually excluded from regulatory trials. Of note, in the -401 study, $60 \%$ (72/120) of patients were elderly (see the table in the ESM) as a post-approval commitment with the European Medicines Agency to assess the safety, tolerability, and efficacy of ESL since this population is underrepresented in clinical trials [15]. Another limitation of this study is the lack of placebo or a comparator group of patients receiving other ASMs to allow direct comparisons. Furthermore, post-marketing data are limited to the reporting rate of events, and the nature and quality of data reported is strongly dependent on the reporter. All these issues limit the quantitative comparison of TEAE and ADR incidences between groups.

\section{Conclusions}

The safety data collected from the seven clinical studies and the 8 years of post-marketing surveillance indicated that no specific safety issues were identified in the treatment of FS with ESL for the elderly patients compared with the non-elderly. The difference in incidences of at least possibly related serious TEAEs reported in elderly versus non-elderly patients (6.7 vs. $2.5 \%$ ) is not unexpected considering the comorbidities and comedications present in elderly patients. ESL is generally safe and welltolerated in elderly populations. In both elderly and nonelderly patients, the most frequently reported at least possibly related TEAEs were dizziness and somnolence in the pooled clinical studies. After 8 years of post-marketing surveillance, and despite the limitations of post-marketing data, the qualitative safety of ESL remains similar to that observed in the clinical studies.

Supplementary Information The online version contains supplementary material available at https://doi.org/10.1007/s40264-021-01097-5.

Acknowledgements The authors thank Franziska T. Arlinghaus Abreu and Dr Phil Nat of Clinipace, Germany, for providing medical writing support/editorial support, including the development of the manuscript, which was funded by Bial-Portela \& $\mathrm{C}^{\mathrm{a}}$, S.A., Coronado, Portugal, in accordance with good publication practice guidelines (http://www. ismpp.org/gpp3).

\section{Declarations}

Funding The sponsor Bial-Portela \& $\mathrm{C}^{\mathrm{a}}$, S.A., Coronado, Portugal, conducted this analysis and was responsible for the study design; the collection, analysis, and interpretation of data; the writing of the report; and the decision to submit the article for publication.
Conflicts of interest Luís M. Magalhães, Raquel Costa, Mariana Vieira, Joana Moreira, Helena Gama, and Patrício Soares-da-Silva are employees of Bial-Portela \& C , S.A.

Availability of data and material Not applicable.

Code availability Not applicable.

Author contributions All authors contributed to the manuscript conception and design. All authors read the draft versions and approved the final manuscript.

Ethics approval All procedures performed in studies involving human participants in all participating countries were in accordance with the ethical standards of the institutional and/or national research committees and with the 1964 Helsinki declaration and its later amendments or comparable ethical standards.

Consent to participate Informed consent was obtained from all individual participants included in the studies reported in this manuscript.

Consent for publication Patients signed informed consent regarding publishing their data

Open Access This article is licensed under a Creative Commons Attribution-NonCommercial 4.0 International License, which permits any non-commercial use, sharing, adaptation, distribution and reproduction in any medium or format, as long as you give appropriate credit to the original author(s) and the source, provide a link to the Creative Commons licence, and indicate if changes were made. The images or other third party material in this article are included in the article's Creative Commons licence, unless indicated otherwise in a credit line to the material. If material is not included in the article's Creative Commons licence and your intended use is not permitted by statutory regulation or exceeds the permitted use, you will need to obtain permission directly from the copyright holder. To view a copy of this licence, visit http://creativecommons.org/licenses/by-nc/4.0/.

\section{References}

1. Motika PV, Spencer DC. Treatment of epilepsy in the elderly. Curr Neurol Neurosci Rep. 2016;16(11):96. https://doi.org/10.1046/j. 1535-7597.2001.00013.x.

2. Wahab A. Difficulties in treatment and management of epilepsy and challenges in new drug development. Pharmaceuticals (Basel). 2010;3(7):2090-110. https://doi.org/10.3390/ph3072090.

3. Kutluay E, McCague K, D'Souza J, Beydoun A. Safety and tolerability of oxcarbazepine in elderly patients with epilepsy. Epilepsy Behav. 2003;4(2):175-80. https://doi.org/10.1016/S15255050(03)00037-4.

4. Romigi A, Femia EA, Fattore C, Vitrani G, Di Gennaro G, Franco V. Zonisamide in the management of epilepsy in the elderly. Clin Interv Aging. 2015;10:931-7. https://doi.org/10.2147/CIA. S50819.

5. CHMP/EWP/566/98 rev 3, Committee for medicinal products for human use. Guideline on clinical investigation of medicinal products in the treatment of epileptic disorders. $2018 \mathrm{https} / / / \mathrm{www} . e m a$. europa.eu/documents/scientific-guideline/draft-guideline-clinicalinvestigation-medicinal-products-treatment-epileptic-disordersrevision-3_en.pdf. Accessed Jun 2021. 
6. Krämer G. Epilepsy in the elderly: some clinical and pharmacotherapeutic aspects. Epilepsia. 2001;42(Suppl 3):55-9. https://doi. org/10.1046/j.1528-1157.2001.042suppl.3055.x.

7. Acharya JN, Acharya V. Epilepsy in the elderly: special considerations and challenges. Ann Indian Acad Neurol. 2014;17(Suppl):S18-26. https://doi.org/10.4103/0972-2327. 128645.

8. Poza JJ. Management of Epilepsy in the elderly. Neuropsychiatr Dis Treat. 2007;3(6):723-8. https://doi.org/10.2147/NDT.S1026.

9. Bergey GK. Initial treatment of epilepsy: special issues in treating the elderly. Neurology. 2004;63(10 Suppl 4):S40-8. https://doi. org/10.1212/WNL.63.10_suppl_4.S40.

10. Gómez-Ibáñez A, Serratosa JM, Guillamón E, Garcés M, Giráldez BG, Toledo M, Salas-Puig J, López-González FJ, RodríguezUranga J, Castillo A, Mauri JA, Camacho JL, López-Gomáriz E, Giner P, Torres N, Palau J, Molins A, Villanueva V. Efficacy and safety of eslicarbazepine-acetate in elderly patients with focal epilepsy: case series. Seizure. 2017;48:53-6. https://doi.org/10. 1016/j.seizure.2017.04.003.

11. Trinka E, Giorgi L, Patten A, Segieth J. Safety and tolerability of zonisamide in elderly patients with epilepsy. Acta Neurol Scand. 2013;128(6):422-8. https://doi.org/10.1111/ane.12162.

12. European Medicines Agency. Zebinix (eslicarbazepine acetate): EU summary of product characteristics. 2020. https://www.ema. europa.eu/en/documents/product-information/zebinix-epar-produ ct-information_en.pdf. Accessed Jun 2021.

13. Sunovion Pharmaceuticals Inc. Aptiom (eslicarbazepine acetate) tablets, for oral use: US prescribing information. 2019. https:// www.accessdata.fda.gov/drugsatfda_docs/label/2019/022416s011 lbl.pdf. Accessed Jun 2021.

14. Gama H, Vieira M, Costa R, Graça J, Magalhães LM, Soaresda-Silva P. Safety profile of eslicarbazepine acetate as addon therapy in adults with refractory focal-onset seizures: from clinical studies to 6 years of post-marketing experience. Drug Saf. 2017;40(12):1231-40. https://doi.org/10.1007/ s40264-017-0576-4.

15. Costa R, Steinhoff B, Gama H, Ikedo F, Rocha JF, Soares-da-Silva P. Safety, tolerability and efficacy of eslicarbazepine acetate as adjunctive therapy in patients aged $\geq 65$ years with focal seizures. Drugs Aging. 2018;35(12):1109-17. https://doi.org/10. 1007/s40266-018-0602-y.

16. Lawthom C, Bermejo P, Campos D, McMurray R, Villanueva V. Effectiveness and safety/tolerability of eslicarbazepine acetate in epilepsy patients aged $\geq 60$ versus $<60$ years: a subanalysis from the Euro-Esli Study. Neurol Ther. 2019;8(2):491-504. https://doi. org/10.1007/s40120-019-0137-0.

17. Andermann E, Rosenfeld W, Penovich P, Rogin J, Cendes F, Carreno $\mathrm{M}$, et al. Comparative analysis of the safety and tolerability of eslicarbazepine acetate in older ( $\geq 60$ years) and younger (18-59 years) adults. Epilepsy Res. 2021;169: 106478. https:// doi.org/10.1016/j.eplepsyres.2020.106478.

18. Elger C, Bialer M, Cramer JA, Maia J, Almeida L, Soares-daSilva P. Eslicarbazepine acetate: a double-blind, add-on, placebocontrolled exploratory trial in adult patients with partial-onset seizures. Epilepsia. 2007;48(3):497-504. https://doi.org/10. 1111/j.1528-1167.2007.00984.x.

19. Elger C, Halasz P, Maia J, Almeida L, Soares-da-Silva P. Efficacy and safety of eslicarbazepine acetate as adjunctive treatment in adults with refractory partial-onset seizures: a randomized, double-blind, placebo-controlled, parallel-group phase III study. Epilepsia. 2009;50(3):454-63. https://doi.org/10.1111/j.1528-1167. 2008.01946.x.

20. Ben-Menachem E, Gabbai AA, Hufnagel A, Maia J, Almeida L, Soares-da-Silva P. Eslicarbazepine acetate as adjunctive therapy in adult patients with partial epilepsy. Epilepsy Res. 2010;89(23):278-85. https://doi.org/10.1016/j.eplepsyres.2010.01.014.

21. Gil-Nagel A, Lopes-Lima J, Almeida L, Maia J, Soares-da-Silva P. Efficacy and safety of 800 and $1200 \mathrm{mg}$ eslicarbazepine acetate as adjunctive treatment in adults with refractory partial onset seizures. Acta Neurol Scand. 2009;120(5):281-7. https://doi.org/10. 1111/j.1600-0404.2009.01218.x.

22. Sperling MR, Abou-Khalil B, Harvey J, Rogin JB, Biraben A, Galimberti CA, Kowacs PA, Hong SB, Cheng H, Blum D, Nunes T, Soares-da-Silva P, 304 Study Team. Eslicarbazepine acetate as adjunctive therapy in patients with uncontrolled partial-onset seizures: results of a phase III, double-blind, randomized, placebocontrolled trial. Epilepsia. 2015;56(2):244-53. https://doi.org/10. 1111/epi.1289.

23. Trinka E, Ben-Menachem E, Kowacs PA, Elger C, Keller B, Löffler K, Rocha JF, Soares-da-Silva P. Efficacy and safety of eslicarbazepine acetate versus controlled-release carbamazepine monotherapy in newly diagnosed epilepsy: a phase III doubleblind, randomized, parallel-group, multicenter study. Epilepsia. 2018;59(2):479-91. https://doi.org/10.1111/epi.13993.

24. Bial-Portela \& Ca, S.A. Data on file; 2017.

25. Lattanzi S, Trinka E, Giovane CD, Nardone R, Silvestrini M, Brigo F. Antiepileptic drug monotherapy for epilepsy in the elderly: a systematic review and network meta-analysis. Epilepsia. 2019;60(11):2245-54. https://doi.org/10.1111/epi.16366.

26. Lattanzi S, Zaccara G, Giovannelli F, Grillo E, Nardone R, Silvestrini M, Trinka E, Brigo F. Antiepileptic monotherapy in newly diagnosed focal epilepsy. A network meta-analysis. Acta Neurol Scand. 2019;139(1):33-41. https://doi.org/10.1111/ane.13025.

27. Werhahn KJ, Klimpe S, Balkaya S, Trinka E, Krämer G. The safety and efficacy of add-on levetiracetam in elderly patients with focal epilepsy: a one-year observational study. Seizure. 2011;20(4):305-11. https://doi.org/10.1016/j.seizure.2010.12. 015.

28. Shorvon SD, Trinka E, Steinhoff BJ, Holtkamp M, Villanueva V, Peltola J, Ben-Menachem E. Eslicarbazepine acetate: its effectiveness as adjunctive therapy in clinical trials and open studies. J Neurol. 2017;264(3):421-31. https://doi.org/10.1007/ s00415-016-8338-2.

29. Jalihal V, Shankar R, Henley W, Parrett M, Tittensor P, McLean BN, Ahmed A, Sander JW. Eslicarbazepine acetate as a replacement for levetiracetam in people with epilepsy developing behavioral adverse events. Epilepsy Behav. 2018;80:365-9. https://doi. org/10.1016/j.yebeh.2018.01.020. 